\title{
Increasing Energy Efficiency in OCDMA Network via Distributed Power Control
}

\author{
Fábio R. Durand, Bruno A. Angélico, \\ Universidade Tecnológica Federal do Paraná, fabiodurand@utfpr.edu.br, \\ bagelico@utfpr.edu.br \\ Taufik Abrão \\ Universidade Estadualde Londrina,taufik@uel.br
}

\begin{abstract}
In this work, we investigate the utilization of transmission power control as mechanism to increase the energy efficiency in optical code division multiplexing access (OCDMA) networks. We have modeled the energy efficiency considering the optical fiber transmission and network infrastructure as encoders, decoders and star coupler. In the analyzed scenario, this model confirms that the energy consumption of the network infrastructure is larger than the energy consumption of the transmission infrastructure; On the other hand, we have proposed a scheme to define the energy efficiency according to the BER level accomplished with the quality of service (QoS) requirements. The main results showed that it is possible to save $70 \%$ of the transmitted energy per bit with the penalty of one order of magnitude of BER.
\end{abstract}

Index Terms - optical code division multiplexing access, distributed power control algorithm.

\section{INTRODUCTION}

The informatics and telecommunications industries have become a component of the economies from the nations. The growing of Internet, information and communications technology (ICT) infrastructures have been showing an exponential behavior and the electrical power consume has increasing in the same way. However, the energy resources available in the world are limited and the energy efficiency in all sort of industry has been encouraged. The energy efficiency in the ICT is represented by the energy consumption per bit of data transported and/or processed [1][2]. Nowadays, the ICT sector is responsible for approximately $5 \%$ of the total electrical power consumption in developed national economies and especially the Internet consumes $1 \%$ of this total electrical power consumption [1]. Recent studies have showed the importance of the consideration of energy consumption in optical communications design, considering the transmission infrastructure (transmitters, receivers, fibers and amplifiers) [3] and network infrastructure (switchers and routers) [4] aspects. In a global scale network, the energy consumption of the switching infrastructure is larger than the energy consumption of the transport infrastructure [2][4]. In this context, it is necessary to improve the energy efficiency of switching and to optimize the network design to reduce the quantity of switching and overheads. Low energy efficiency is observed mainly at access network, because the access equipment consumes almost 50\% of energy consumed in 
the core or metro equipment [3]. However, the amount of bits transported in core or metro network is larger than in the access network.

Optical-code-division multiple access (OCDMA) based networks [5] have attracted a lot of interests due to various advantages including asynchronous operation, high network flexibility, protocol transparency, simplified network control and potentially enhanced security [5-9]. In OCDMA each different code defines a user and different code-users can share a common channel. In a common channel, the interference that may arise between different code-users is known as multiple access interference (MAI) and can limit the number of code-users utilizing the channel simultaneously. OCDMA can be divided into non-coherent (unipolar) and coherent (bipolar) systems. The non-coherent systems are based only on intensity modulation of optical power [7], and coherent systems are based on modulation of amplitude and phase [8]. The coherent code is true-orthogonal and non-coherent code is pseudo-orthogonal. As a consequence, the performance of coherent codes is higher than noncoherent codes when analyzing the signal-to-interference plus noise ratio (SINR) [8]. However, the main drawback of coherent OCDMA lies in the technical implementation difficulties, concomitant with the utilization of phase-shifted optical signals [6-8]. The non-coherent codes can be classified in one dimensional (1-D) and two dimensional (2-D) codes. In 1-D codes, the bits are subdivided temporally into many short chips with a designated chip pattern representing a user's code. On the other hand, in 2-D codes the bits are subdivided into individual time chips, and each chip is assigned to an independent wavelength from a discrete set of wavelengths [9]. The 2-D codes have better performance than 1-D codes and can significantly enhance the number of active and potential users [9]. In the OCDMA, the nearfar ratio increases the multiple access interference effects, because each active node in transmission mode contributes with MAI and the power penalty varies dynamically. Therefore, the static power budget design does not solve this problem [10][11]. Furthermore, if the distances between the nodes are quite different, as would be the case in practice, the power received from different nodes will be significantly different. Thus, considering as reference one OCDMA node, the performance of closer nodes is many orders of magnitude better than that of far nodes.

Hence, an efficient power control is needed to overcome this problem and to enhance the performance and throughput of the optical network [12]. This could be achieved via signal-tointerference (SINR) optimization [12][13]. In this case, analogous to a wireless CDMA cellular system, the power control, centralized or distributed, is one of the most important issues since it has a significant impact on both performance and capacity; it is the most effective way to avoid the near-far problem and to increase capacity. Previously, this problem has been investigated in OCDMA networks to solve the near-far problem [10] and to obtain quality of service (QoS) at physical layer [11]; however this issue has not been investigated with focus on the energy efficiency.

In this work, we study the utilization of distributed power control as mechanism to obtain energy efficiency improvement in OCDMA access network with non-coherent 2 -D codes. The performance of this architecture networks is analyzed considering the loss characteristics of encoders, decoders and star coupler. Our objective consists in investigating the viability of 
power control in order to increase the energy efficiency of the OCDMA network and to determine the best tradeoff between the energy efficiency and near-far mitigation.

This paper is organized as following: Section II describes the architecture of the network for 2-D codes. Section III discusses the proposed methodology for increasing OCDMA energy efficiency. The performance of proposed methodology systems is developed in Section IV. Section V shows representative numerical results for the proposed approach; finally, in Section VI the main conclusions are presented.

\section{NETWORK ARCHITECTURE}

The OCDMA architecture considered in this work is formed by $K$ nodes interconnected by passive star coupler, in a broadcast-and-select pattern as shows Fig. 1. For viability characteristics, we consider network equipment, such as code-processing devices (encoders and decoders at the transmitter and receiver) and star coupler. Such devices could be made using robust, lightweight, and low-cost technology platforms with commercial-off-the-shelf technologies [14], [15].

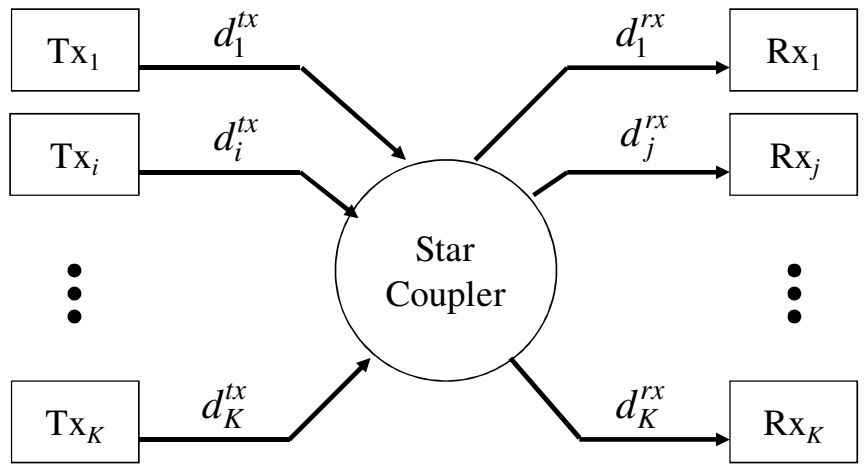

Fig. 1. OCDMA network architecture.

The transmitting and receiving nodes create virtual path based on the code and the total link length is given by $d_{i j}=d_{i}^{t x}+d_{j}^{r x}$, where $d_{i}^{t x}$ is the link length from the transmitting node to the star coupler and $d_{j}^{r x}$ is the link length from the receiving node to the star coupler. The received power at a $j$ th node is given by $P_{r j}=a_{s t a r} p_{i} \exp \left(-\alpha_{f} d_{i j}\right)$, where $p_{i}$ is the transmitted power by $i$-th transmitter node, $\alpha_{f}$ is the fiber attenuation $\left(\mathrm{km}^{-1}\right)$ and $a_{\text {star }}$ is the star coupler attenuation (linear units). Considering deciBell units, $a_{\text {star }}=10 \log (K)-\left[10 \log _{2}(K) \log _{10} \delta\right]$, where, $\delta$ is the excess loss ratio.

The 2-D wavelength-hopping/ time-spreading code sequence is illustrated in Fig. 2. This 2-D code is transmitted and its destiny in the network is determined by a particular code sequence. The 2-D codes can be represented by $N_{\lambda} \times N_{T}$ matrices, where $N_{\lambda}$ is the number of rows, that is equal to the number of available wavelengths, and $N_{T}$ is the number of columns, that is equal to the code length. The code length is determined by the bit period $T_{B}$ which is subdivided in small units called chips, each of duration $T_{c}=T_{B} / N_{T}$. In each code, there are $W$ short pulses of different wavelength, where $w$ is called the weight of the code. An $\left(N_{\lambda} \times N_{T}, W\right.$, $I_{a}, I_{c}$ ) code is the collection of binary $N_{\lambda} \times N_{T}$ matrices each of code weight $w ; I_{a}$ and $I_{c}$ are 
nonnegative integers and represent the constraints on the autocorrelation and crosscorrelation [8], respectively.

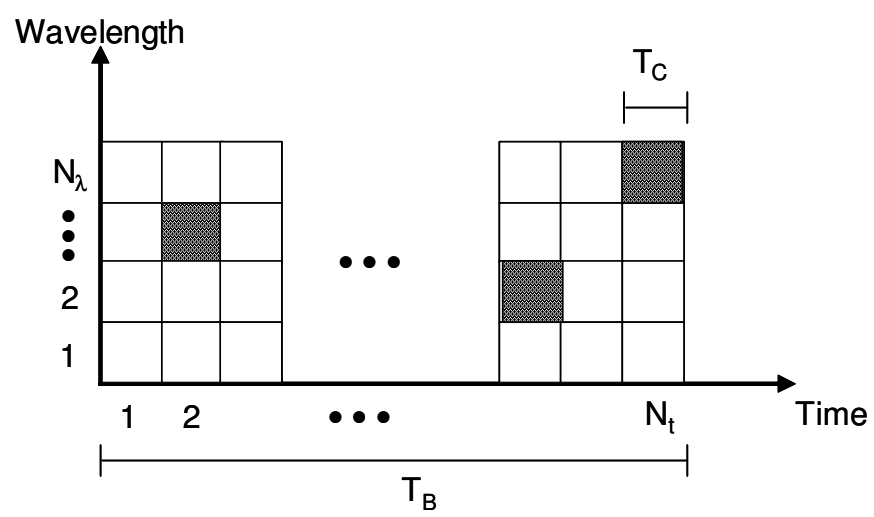

Fig. 2. Schematic of 2D wavelength-hopping time spreading code sequence.

The 2-D OCDMA utilizes multi-wavelength sources such light-emitting diodes, amplified spontaneous emission noise from erbium-doped fiber amplifier (EDFA), gain switched FabryPérot lasers, and supercontinuum generation [15]. These sources avoid the need for rapidly wavelength hop according the wavelength-hopping pattern. The encoder essentially creates a combination of two patterns: a wavelength-hopping pattern and a time-spreading pattern. The common technology applied in encoders/decoders with delay lines are array waveguide gratings (AWGs), thin-film filters (TFFs), simultaneously as fiber Bragg gratings (FBGs), holographic Bragg reflectors (HBRs), chirped Moire gratings (CMGs) [16][17]. The schemes of encoders commonly utilized are based on AWG, TFF and FBGs, as are showed in Fig. 3.

The losses associated with the encoders/ decoders are given by [15][16][17]

$$
\begin{gathered}
C_{A W G}(d B)=2 a_{A W G}+a_{\text {Delay }} \\
C_{T F F}(d B)=6 \log _{2}\left(N_{\lambda}\right)+a_{T F F}+a_{\text {Delay }} \\
C_{\text {Bragg }}(d B)=N_{\lambda} a_{\text {Bragg }}+a_{\text {Cirulator }}
\end{gathered}
$$

where $a_{A W G}$ is the AWG loss, $a_{\text {Delay }}$ is the delay line loss, $a_{\text {TFF }}$ is the TFF loss, $a_{B r a g g}$ is the FBG loss and $a_{\text {Cirulator }}$ is the circulator loss. The loss usual value for these equipments are $a_{A W G}=2.5 \mathrm{~dB}, a_{\text {Delay }}=1 \mathrm{~dB}, a_{T F F}=0.5 \mathrm{~dB}, a_{\text {Bragg }}=0.5 \mathrm{~dB}$ and $a_{\text {Cirulator }}=3 \mathrm{~dB}$. The AWG encoder has approximately uniform loss $(6 \mathrm{~dB})$ independently of the number of wavelengths $\left(N_{\lambda}\right)$ [16]. For this characteristic, we will consider the AWG as encoder/decoder in the rest of this work. 


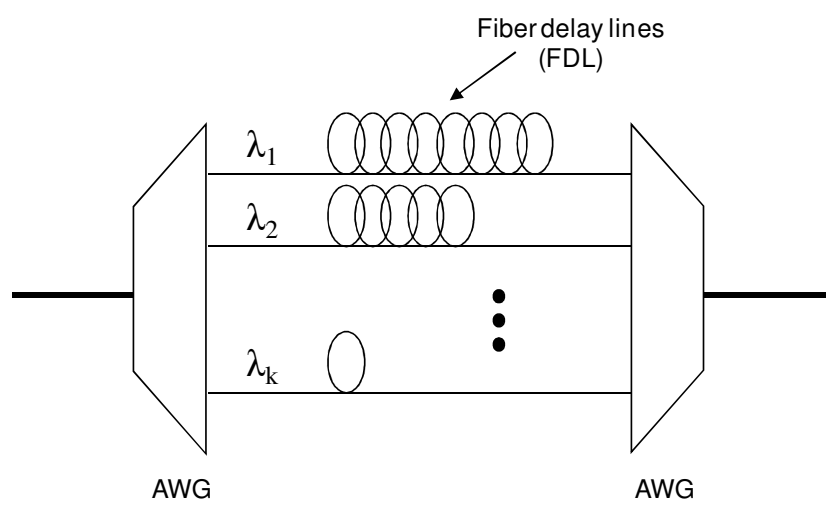

(a)

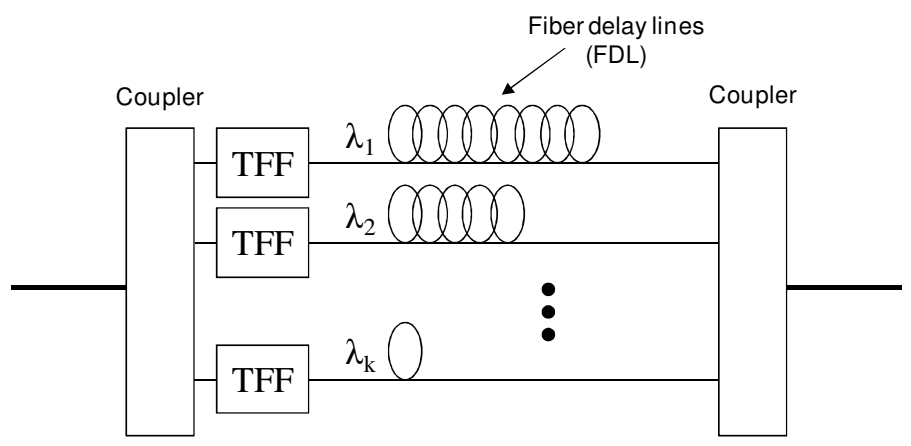

(b)

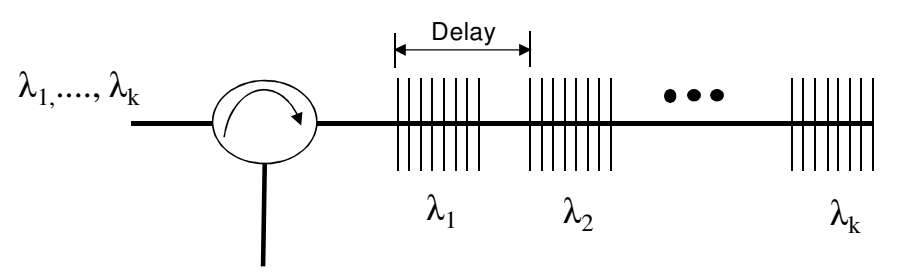

(c)

Fig. 3. Schematic of 2-D encoders and decoders (a) Array waveguide gratings (AWGs), (b) Thinfilm filters (TFFs), and (c) fiber Bragg grating (FBG).

\section{THEORETICAL ANALYSIS}

\section{a. Energy efficiency and power allocation}

The necessary energy in each $i$ th node for the transmission of 1 bit can be expressed as [3],

$$
E_{i}=p_{i} T_{b i t} \quad[\mathrm{~J} / \text { bit }], \quad i=1, . ., K
$$

where $K$ is the number of nodes, $T_{b i t}$ is the time to transmit one bit over the network given by $T_{b i}=1 / R$, where $R$ is the bit rate (bits/seconds) and $p_{i}$ is the transmitted power. In order to determine the energy per bit values it is necessary to define the individual node transmitting power $\left(p_{i}\right)$. The $p_{i}$ is obtained by power budget or power control and it is associated to a QoS, a SINR and a maximum bit error rate (BER) tolerated by the optical nodes. Under a power control situation, each optical node adjusts its transmitting power in an attempt to maximize the number of transmitted bits with minimum energy consumption. This concept can be formulated by the energy efficiency definition [18], 


$$
\varepsilon_{i}=R \frac{L}{M} \frac{f\left(\gamma_{i}\right)}{p_{i}}, \quad i=1, . ., K
$$

where $M$ is the number of bits in each transmitted packet, $L$ is the number of information bits contained in each data packet and $f\left(\gamma_{i}\right)$ is the efficiency function, which approximates the probability of error-free packet reception. It can be approximated by $f\left(\gamma_{i}\right)=\left(1-B E R_{i}\right)^{M}$, with $y_{i}$ being the SINR for the $i$ th node, given by [14][18],

$$
\gamma_{i} \propto \frac{g_{i i} p_{i}}{I_{i}+N_{i}}, \quad i=1, . ., K
$$

where $g_{i j}$ are the total loss in the path that connects $i$ th-Tx node to $j$ th-Rx node, $I_{i}$ is the total interference power level comes from the others transmitters nodes and $N_{i}$ is the receiver power noise level. Both terms $I_{i}+N_{i}$ will be described in details in the next subsection, eq. (8).

In the same way this concept is used to define a metric named utility that is the number of bits received per energy expended or the relation of the throughput and power dissipation [18]. Hence, for each $i$ th node, the maximum number of transmitted bits occurs at power level for which the partial derivative of $\varepsilon_{i}$ with respect to $p_{i}$ equals to zero, $\partial \varepsilon_{i} / \partial p_{i}=0$. So, the derivative of energy efficiency can be obtained referring to efficiency function $f\left(\gamma_{i}\right)$ and eq. (4), setting:

$$
\frac{\partial \varepsilon_{i}}{\partial p_{i}}=\frac{R}{p_{i}^{2}} \frac{L}{M}\left(\gamma_{i} \frac{\partial f\left(\gamma_{i}\right)}{\partial \gamma_{i}}-f\left(\gamma_{i}\right)\right), \quad i=1, . ., K
$$

From (5) assuming $p_{i}>0$, the necessary condition to maximize the energy efficiency is immediately obtained:

$$
\gamma_{i} \frac{\partial f\left(\gamma_{i}\right)}{\partial \gamma_{i}}-f\left(\gamma_{i}\right)=0, \quad i=1, . ., K
$$

So, adopting the widely accepted approximation for BER performance with uncoding system $(M=L)$ and binary modulation, the bit error rate can be approximated as $B E R_{i}=e^{-\gamma_{i}}$. Hence, we have: $\partial f\left(\gamma_{i}\right) / \partial \gamma_{i}=\partial / \partial \gamma_{i}\left(1-e^{-\gamma_{i}}\right)=e^{-\gamma_{i}}$. As a result, we can conjecture that the optimum power allocation criterion in terms of energy efficiency is given by

$$
p_{i}=\kappa \cdot \frac{I_{i}+N_{i}}{g_{i i}} \cdot e^{\gamma_{i}} \cdot\left(1-e^{-\gamma_{i}}\right)^{M}, \quad i=1, . ., K
$$

where $\mathcal{K}$ is a constant of proportionality. Besides, further simplification can be obtained if the condition $M=1$ could be assumed:

$$
p_{i}=\kappa \cdot\left(e^{\gamma_{i}}-1\right) \cdot \frac{I_{i}+N_{i}}{g_{i i}}, \quad i=1, . ., K
$$

\section{b. Proposed scheme}

Note that in order to satisfies (6) it is necessary adjust the SINR at each received node equals to the target SINR, $\gamma_{i}=\gamma_{i}^{*}$. In this context, we propose the utilization of power control iterative algorithm in order to establish the lower energy per bit according to the OCDMA 
network QoS requirements. Fig. 4 shows the flowchart with the adopted scheme to assign the energy per bit transmitted in each node. This scheme aims to save energy per bit at each node and, as a consequence, decreases the total energy consumption overall the network.

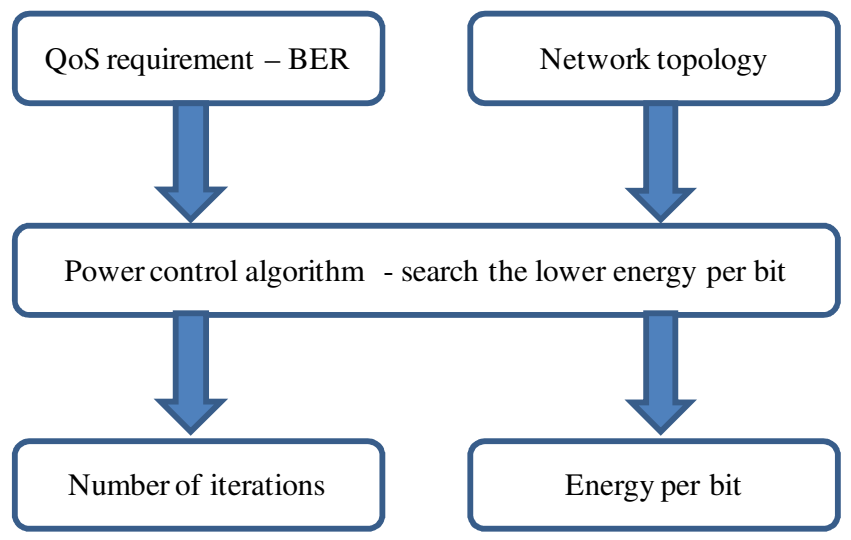

Fig. 4. Flowchart with the proposed scheme to save energy per bit.

This scheme is based on the power control with the restriction of QoS requirement based on BER level. The entries are the BER level and the network topology, comprising the following parameters: node distances, links lengths, fiber parameters, EDFA preamplifiers value, OCDMA code parameters, and so forth.

The iterative power control algorithm defines the transmitted power according to the number of active transmitting nodes aiming to establish individual target value of BER, and taking into account as low level energy per bit as possible. The outputs of the scheme are the necessary number of iterations of the iterative power control algorithm and the optimized value of energy per bit necessary for each node. In the next subsections we will illustrate BER computation, the main characteristics of the power control procedure, as well as the way to apply the power control in order to obtain a more energy efficient OCDMA network.

\section{Performance Evaluation}

\section{a. Signal-to-interference plus noise ratio (SINR) and Bit error rate (BER)}

In [11], Inaty et al. derive an expression for optical SINR in a multirate chip-synchronous 2D-OCDMA system. Assuming single-rate case, the SINR for the $i$ th node of a 2 -D code OCDMA system can be precisely written as:

$$
\gamma_{i}=\frac{N_{T}^{2} g_{i i} p_{i} G_{a m p}}{\sigma^{2} G_{a m p} \sum_{j=1, j \neq i}^{K} g_{i j} p_{j}+2 P_{N}}, i=1, . ., K
$$

where $g_{i j}$ and $G_{a m p}$ are, respectively, the total loss and gain of the preamplifier in the path that connects $i$ th-Tx node to $j$ th-Rx node. $\sigma^{2}$ is the average variance of the Hamming aperiodic cross-correlation amplitude [8], $P_{N}$ is the spontaneous amplified emission (ASE) noise power in the preamplifier considering the two polarization mode presented in a single mode fiber 
and $p_{j}$ is the other nodes transmitted power. Note that the usual receiver noise power includes thermal noise, shot noise and optical preamplifier noise. However, ASE in the optical preamplifier will be the main limiting factor (in addition to the MAI), compared to thermal and shot noise at the receiver [10]. In our work, the receiver noise power is represented by

$$
P_{N}=n_{S P} h f(G-1) B_{o}
$$

where $n_{s p}$ is the spontaneous emission factor, typically around 2 to $5, h$ is Planck's constant, $f$ is the carrier frequency, $G$ is the amplifier gain and $B_{o}$ is the optical bandwidth. Ideally, in order to reduce the ASE noise power, the optical bandwidth can be set to a minimum of $B_{o}=$ $2 R$. Assuming Gaussian noise approximation, the BER associated with the $i$-th node is related to its SINR by $P_{b, i}=\operatorname{erfc}\left(\sqrt{\gamma_{i}} / 2\right)$, where $\operatorname{erfc}($.) is the complementary error function.

\section{b. Power control}

The power budget analysis establishes the transmitted power necessary to reach the photodiode sensibility. The power budget in dB must satisfy the follow inequality,

$$
p_{s} \leq p_{i}+G_{a m p}-C_{E n c}-\alpha_{f} d_{i j}-a_{s t a r}-C_{D e c}-N_{M A I} \quad[\mathrm{~dB}], \quad i 1, . ., K
$$

where $p_{s}$ is the receiver power sensibility, $N_{M A I}$ is the MAI interference variance, $C_{E n c}$ is the encoder losses and $C_{\text {Dec }}$ is the decoder losses. The losses associated with the encoders/ decoders are given by (1).

We observe in (10) the static characteristics of the power budget design, mainly because the MAI varies with the number of active nodes transmitting simultaneously. The design based on static power budget results in a transmitting power higher or lower than the necessary; as a result, an increase in the near-far problem occurs. To solve this problem, it is adequate to apply dynamic power control that defines the transmitting power according the number of active transmitting nodes.

The power control in optical networks is an optimization problem aiming to establish individual target value of SINR, denoted by $\gamma_{i}^{*}$; hence, each node unit transmitting with power $p_{i}$, has to be controlled in order to satisfy the relation $\gamma_{i} \geq \gamma_{i}^{*}$. The maximum achievable SINR at the receiving nodes is equal to the maximum achievable carrier to interference ratio (CIR) at the receiver output times $\mathrm{N}_{T}^{2} / \sigma^{2}$. Denoting $\Gamma_{i}$ as the CIR at the required decoder input, in order to get a certain QoS which is associated to a maximum tolerable BER at the $i$ th optical node, and defining the $K$-dimensional column vector of the transmitted optical power $\mathbf{p}=\left[p_{1}\right.$, $\left.p_{2}, \ldots \ldots p_{K}\right]^{T}$, then the optical power control optimization problem consists in finding the optical power vector $\mathbf{p}^{*}$ that minimizes the following cost function [19]:

$$
J(\mathbf{p})=\mathbf{1}^{T} \mathbf{p}=\sum_{\mathrm{i}=1}^{\mathrm{K}} p_{i}
$$

subject to the constraints: 


$$
\begin{gathered}
\Gamma_{i}=\frac{g_{i i} p_{i} G_{a m p}}{G_{a m p} \sum_{j=1, j \neq i}^{K} g_{i j} p_{j}+2 P_{N}} \geq \Gamma^{*} \\
P_{\min } \leq p_{i} \leq P_{\max } \quad \forall i=1, . ., K
\end{gathered}
$$

where $\mathbf{1}^{T}=[1, \ldots, 1]$ and $\Gamma^{*}$ is the minimum CIR to achieve a desired QoS. The elements gij that represent the connections of transmitter-receiver pairs constitute the network attenuation matrix:

$$
\mathbf{G}=\left[\begin{array}{cccc}
g_{11} & g_{12} & \cdots & g_{1 K} \\
g_{21} & g_{22} & \cdots & g_{2 K} \\
\vdots & \vdots & \ddots & \vdots \\
g_{K 1} & g_{K 2} & \cdots & g_{K K}
\end{array}\right]
$$

Using matrix notations, (12) can be written as,

$$
\left[\mathbf{I}-\Gamma^{*} \mathbf{H}\right] \mathbf{p} \geq \mathbf{u}
$$

where $\mathbf{I}$ is a $K$-dimension identity matrix, $\mathbf{H}$ is the normalized interference matrix, whose elements can be evaluated by

$$
h_{i j}= \begin{cases}0, & i=j, \\ \frac{g_{i j}}{g_{i i}}, & i \neq j,\end{cases}
$$

and the th element of vector $\mathbf{u}$ is given by:

$$
u_{i}=\frac{\Gamma^{*} P_{N}}{G_{a m p} g_{i j}}
$$

Note that in (15) we have a scaled version of the noise power. Solving (14), substituting inequality by equality, we get the optimized power vector solution through matrix inversion:

$$
\mathbf{p}^{*}=\left[\mathbf{I}-\Gamma^{*} \mathbf{H}\right]^{-1} \mathbf{u}
$$

This optical power vector represents the case of power equilibrium at the receiver node, and is the optimal power required achieving the target CIR. Increasing the value of the CIR would result in higher optical power values that could result in a maximum power higher than the allowed. In this case, a solution would be fixing or decreasing the target CIR value or removing (switching off) some users from the network. The centralized power control is obtained by matrix inversion, as illustrated in (17), and corresponds to an existence of a central node. The central node storages information about all physical network architecture like fiber length between nodes, amplifier position, regular updating about transmission establishment and dynamic of the optical traffic. These characteristics are the drawback of centralized control [10][19]. On the other hand, the distributed power control algorithm (DPCA) synthesis consists of the development of a systematic procedure for the vector $\mathrm{p}$ evolution in order to reach the optimum value, $\mathbf{p}^{*}=\left[\mathbf{I}-\Gamma^{*} \mathbf{H}\right]^{-1} \mathbf{u}$ based on the $\gamma_{i}^{*}, \gamma_{i}$ and $p_{i}$ values. The optimum solution for the power allocation problem satisfies the following iterative process [14][19] (For more details about the derivation of (18), see Appendix I): 


$$
p_{i}[n+1]=p_{i}[n]-\alpha\left(1-\frac{\gamma_{i}^{*}}{\gamma_{i}[n]}\right) p_{i}[n], i=1, . ., K
$$

where $n$ is the number of iterations and $\alpha$ is the convergence. Note that the convergence factor $\alpha$ in (18) is the numerical integration step to solve an ordinary differential equation, which with some minor alterations is also considered in many other studies of power control [10][14][19]. The algorithm represented by eq. (18) was evaluated for positive and no greater than 1 value of $\alpha$, since it was shown in [19] that the algorithm divergence outside this interval. This parameter is responsible for the convergence speed: values close to 1 indicate fast convergence; however, the quality of the solution after convergence is not excellent when compared with values close to 0 [14]. There are more details about several aspects that we do not discuss here, such as, convergence, proximity to the optimum value, and sensibility to estimation errors as well, that are discussed in details in [14].

Observing the scalar equations in (18) we can infer that the transmitted power actualization of each node depends only on its own parameters. This apparent uncoupling among nodes follows from the fact that we are estimating the SINR directly and not calculating it from the equation (8). It is obvious that for obtaining the SINR from (8) it would demand an expensive procedure in order to estimate all channel gains (interferers and desired user), imposing a centralized implementation of the DPCA, and finally increasing the algorithm implementation complexity. So, we must avoid this approach. However, the recursion in eq. (18) can be effectively implemented in each ( $i$ th) optical node because all necessary parameters (i.e., $\alpha_{i}$, the QoS level given by $\gamma_{i}^{*}$, and the transmitted power $p_{i}[n]$ ), except $\gamma_{i}[n]$, can be considered known at the $i$ th node. Under the distributed power control approach, the SINR $\gamma_{i}[n]$ is obtained at the correspondent destination optical node that demodulates the signal from the optical user $i$ th. In this way, the destination optical node estimates $\gamma_{i}[n]$, quantizes it in a

convenient number of bits, and transmits this information to the optical user $i$ th through a return channel. Indeed, it is possible to measure $\gamma_{i}[n]$ without the effective knowledge of the

information from all the optical interferer nodes, only sensing the $\dot{r}$ th optical signal for a period of time at the destination optical node, following by a sensing of all (sum) interferer signals (in the absence of $i$ th transmitted signal) in a second period of time. In summary, (18) depends only on local parameters allowing the power control to work in a distributed manner, i.e., each one of the $K$ links would be able to carry out separately the respective power control procedure. This brief explanation justifies the name distributed power control algorithm (DPCA) for the set of recursions implicit in (18).

To cope with the SINR optimization, the minimum power constraint (which is also called sensitivity level) assures detection of the optical signal by all optical devices. On the other hand, the maximum power constraint guarantees the minimization of nonlinear physical impairments, because it makes the aggregated power on a link to be limited to a maximum value.

The convergence characteristics of the transmitted power given by DPCA present an asymptotic behavior $\lim p(n)=p^{*}$ for any strictly positive initial condition $\left(p_{i}>0\right)$ [19]. $n \rightarrow \infty$

Therefore, the energy efficiency presents the same asymptotic behavior of the transmitting 
power, and then an increase in the number of iterations results in the convergence for the transmitting energy [18].

\section{NUMERICAL RESULTS}

The scenario considered in our study was presented in Fig. 1. The adopted distances between Tx nodes and star coupler are shown in Fig. 5.a, while distances between Rx nodes and star coupler considering 31 nodes are presented in Fig. 5.b. The nodes are uniformly distributed over an area with a radius between $2 \mathrm{~km}$ and $50 \mathrm{~km}$; hence, the range of the total link length is within $[4 ; 100] \mathrm{km}$. We consider the transmission of an OCDMA signal over a nonzerodispersion-shifted (NZD) fiber (ITU-T G.655) with attenuation $\alpha_{f}=0.2 \mathrm{~dB} / \mathrm{km}$ and non-linear parameter $\Gamma=2(\mathrm{~W} . \mathrm{km})^{-1}$ utilizing AWG encoders/decoders. Applying typical parameters values: $n_{S P}=2, h=6.63 \times 10^{-34} \mathrm{~J} / \mathrm{Hz}, f=193.1 \mathrm{THz}, G=20 \mathrm{~dB}, B_{o}=30 \mathrm{GHz}$, immediately we obtain ASE power noise $P_{N}=15 \times 10^{-7} \mathrm{~A}^{2}$. The transmission rate considered is 2.5 Gbps, SINR target $\gamma_{i}^{*}=27 \mathrm{~dB}$ for 2 -D codes $(4 \times 101,4,1,0)$.

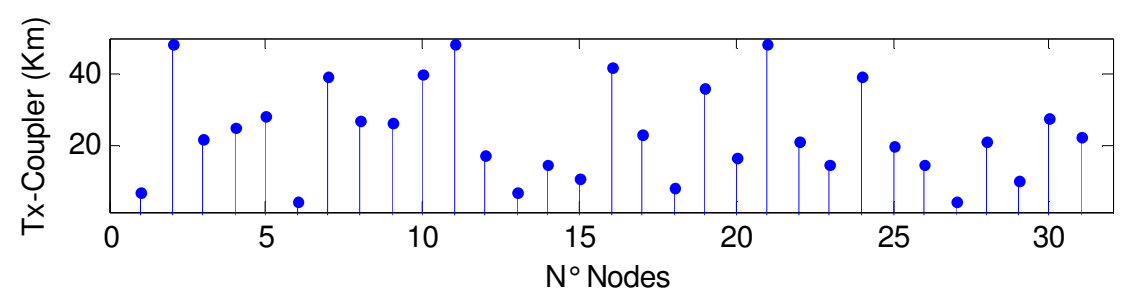

(a)

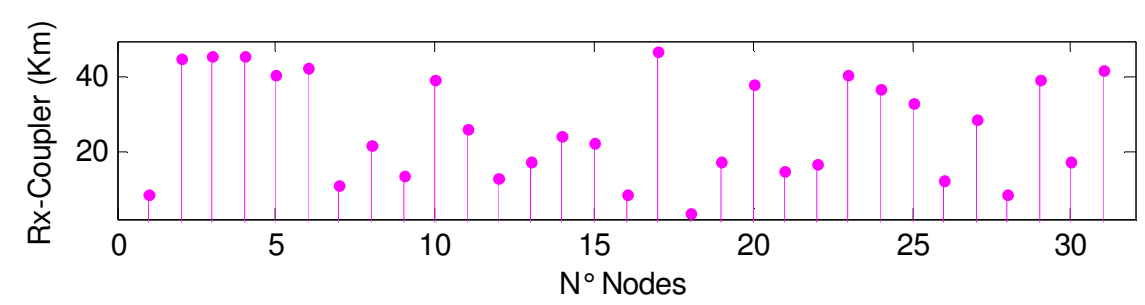

(b)

Fig. 5. a) Distance between Tx nodes and star coupler; b) Distance between Rx nodes and star coupler.

Fig. 6.a and 6.b present the energy per bit assignment per node for OCDMA system considering only the transmission infrastructure (transmitters, receivers, fibers and amplifiers) and transmission plus network infrastructure (encoders, decoders and switchers), respectively.

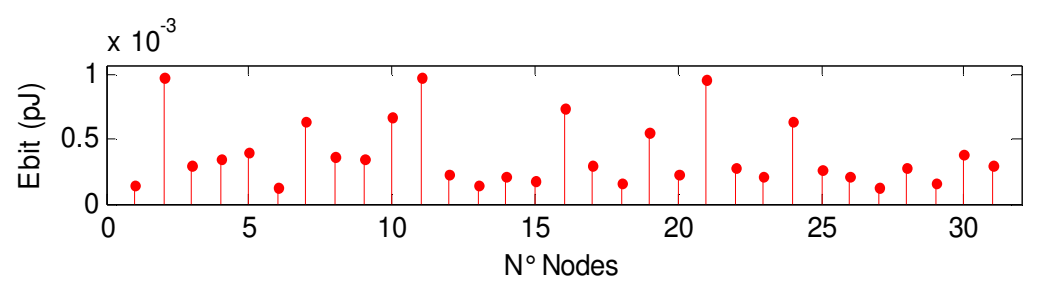

(a) 


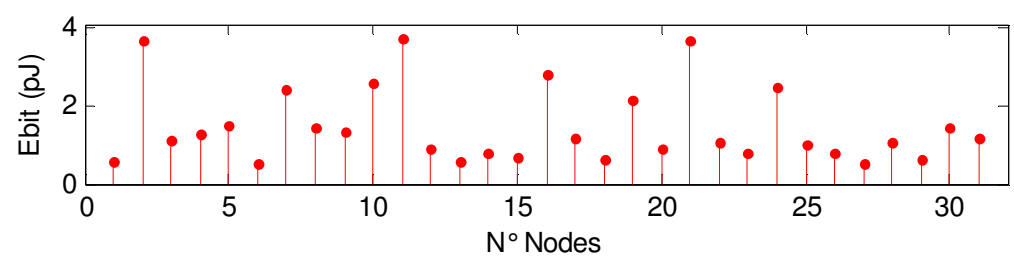

(b)

Fig. 6. Energy per bit assignment per node: a) Transmission infrastructure; b) Transmission plus network infrastructure.

As one can observe from Fig. 6.a and 6.b, there is a considerable difference between the values of energy per bit per node considering only the transmission infrastructure and considering the overall system, i.e. aggregating losses and multiple access interference in transmission plus network. Indeed, in accordance with [3], [4], our numerical results show the lower energy per bit necessary for the transmission system even we do not use optically amplified links and our system adopt only EDFA preamplifier. The losses due to the OCDMA encoder/decoder, as well as the loss introduced by the star coupler, have been resulted in a substantial difference of three orders of magnitude in the energy per bit regards to other conventional OCDMA systems with same parameters considered herein. This result is confirmed by static power budget considering losses of $6 \mathrm{~dB}$ for encoder, $6 \mathrm{~dB}$ for decoder and $14 \mathrm{~dB}$ for star coupler. These numerical results for the energy per bit were obtained applying the centralized control strategy, i.e. solving (17) by matrix inversion. However, the drawback of centralized control is the necessity of central node with parameters knowledge of the physical network architecture.

Fig. 7 shows the energy per bit per node as a function of the number of iterations for the classical iterative DPCA [19] with $\alpha=0.8$, considering the same scenario (transmission and network infrastructure) presented in Fig. 5. This adopted value for the convergence velocity factor allows to combine a relative fast convergence velocity with acceptable quality of the solution after 40-50 iterations [13][14]. A decrease of 75\% in the number of iterations required to convergence was observed in [13] when the value of $\alpha$ increase from 0.2 to 0.8 . Indeed, from Fig. 7, one can see when iteration evolving, the energy per bit per node of the DPCA approaches the values calculated by centralized control strategy, eq. (7), represented by horizontal dot lines. Furthermore, we observe an increase in the energy per bit per node with the number of iterations and this characteristic could be utilized to control the energy efficiency. In our proposed scheme, the number of iterations is the parameter in which the transmitted energy per bit per node can be controlled according to the SINR penalty. However, the SINR penalty affects the BER and could result in an unrealistic OCDMA system. This issue is directly or indirectly considered in Figs. 8 and 9. 


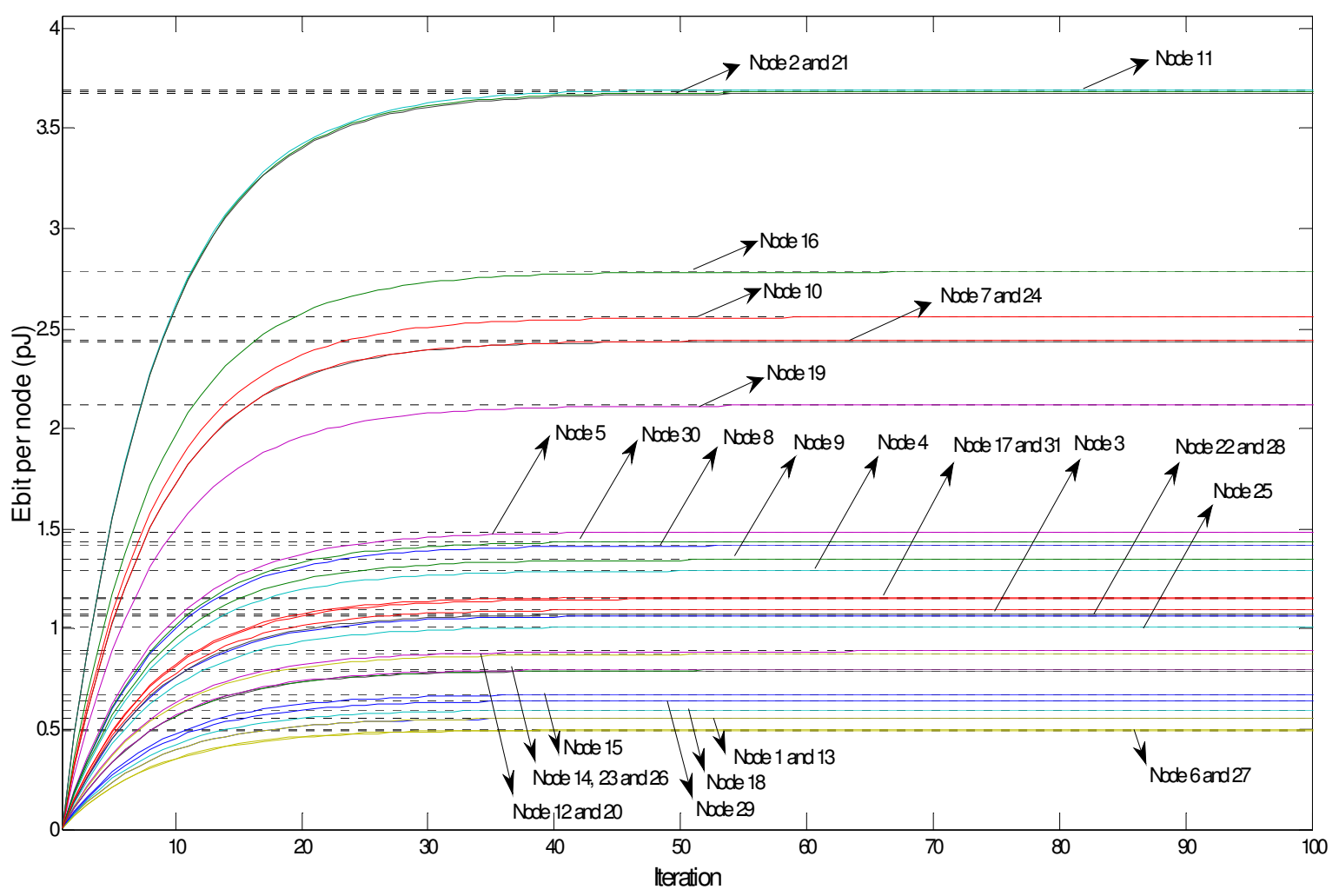

Fig. 7. Convergence behavior in terms of energy per bit per node versus the number of iterations for the classical DPCA, considering the same scenario from Fig. 5, and $\alpha=0.8$.

Fig. 8 shows the BER performance as a function of the number of active optical nodes for a code weight of 4 and a code length of 101, applying the proposed distributed DPCA scheme. The scenario is the same adopted in Fig. 5 with $\alpha=0.8$. The BER system with DPCA considering different percentage of energy per bit of $5 \%, 27 \%$ and $100 \%$ is obtained; these percentages are obtained with, 3, 12 and 60 iterations, respectively. Note that in this scenario, 100\% energy per bit is achieved after 60 iterations (total DPCA convergence), in a similar way showed in Figure 7. 


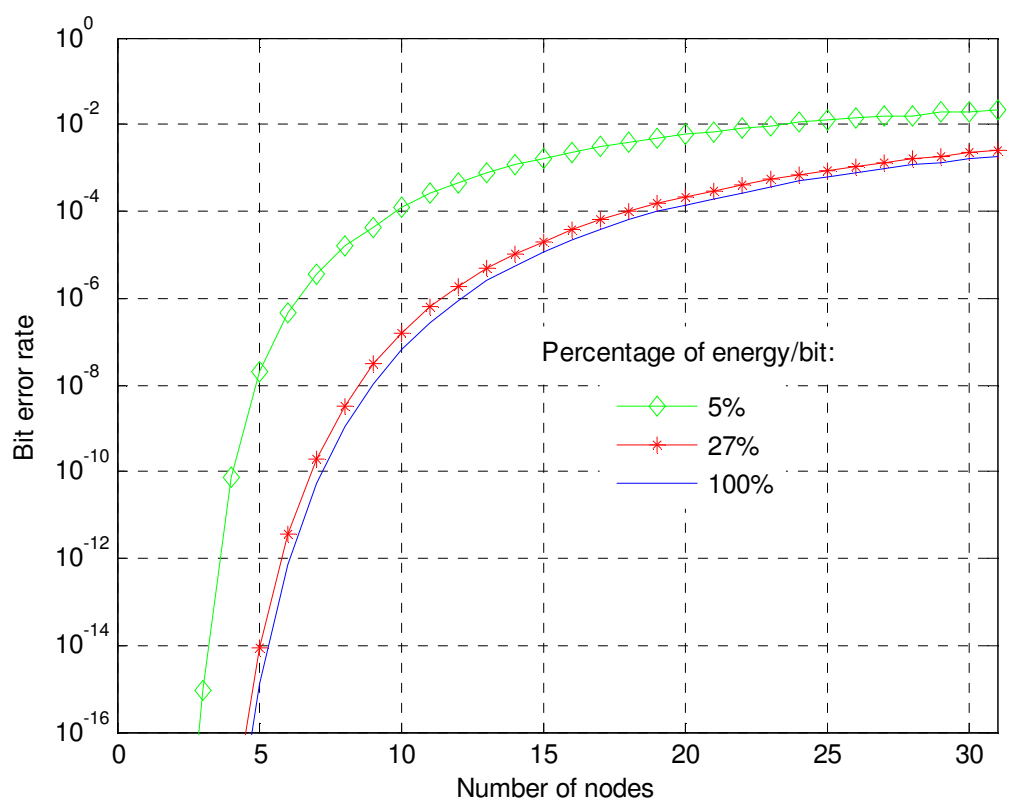

Fig. 8. BER against active nodes for the code weight of 4 and code length of 101 applying the proposed scheme with DPCA power control with percentage of energy per bit of $5 \%, 27 \%$ and $100 \%$, obtained with 3, 12 and 60 iterations, respectively.

It can be seen from Fig. 8 the impact of power control procedure (in terms of transmitting energy per bit) on the bit error rate performance. The deployment of DPCA with transmission of $100 \%$ of energy per bit approaches the BER considering the ideal system [10], i.e. equal distance for all nodes. Indeed, with lower energy per bit (5\%), the SINR declines because the MAI and near-far effects are strongly influenced by an increase in the number of active nodes. Under this situation, an error occurs when cross-correlational pulses from the $(K-1)$ interfering reaches a level higher than the autocorrelation peak, changing a bit zero to a bit one. As a consequence, under the non-optimized SINR, the bits are more vulnerable to distortion, which reduces the likelihood of their successful reception. It is worth mentioning that with the utilization of only $27 \%$ of the maximal energy per bit, the BER performance approaches the result with 100\% of energy per bit. Besides, the error-free limit (BER=10-9) is exceeded when more than 4 or 8 nodes are actives with $5 \%$ or $100 \%$ of the energy per bit, respectively.

On the other hand, a forward error correction (FEC) limit of $10^{-5}$ is exceeded when more than 8 or 14 optical nodes become actives with $5 \%$ or $100 \%$ of the energy per bit, respectively. However, the FEC scheme presents high energy consumption of $250 \mathrm{pJ} / \mathrm{b}$ with low energy efficiency, so that it is difficult to perform at high bit rates [20]. In this context, further studies including FEC schemes are necessary in order to evaluate the global energy efficiency impact on the OCDMA system.

Fig. 9 presents the BER performance against the percentage of transmitting energy per bit for the same scenario adopted in Fig. 8. We show the BER with DPCA power control for the number of active nodes of 5, 10 and 15. As expected, the low percentage of energy per bit results in a high BER, mainly for higher number of active nodes. Furthermore, one can control 
the optical BER level performance, according the system requirement, by adjusting the percentage of energy per bit transmitted. Thus, there is a trade-off between transmitting energy and BER. For example, with 5 active nodes it is possible to obtain a BER level of $10^{-9}$ with $9 \%$ of the energy per, which results in $91 \%$ of reduction in the energy consumption when compared with a system without any energy control mechanism. Furthermore, the general behavior of this figure suggests that it is possible to save up to $70 \%$ of energy per bit transmitted with the penalty of one order of magnitude of BER.

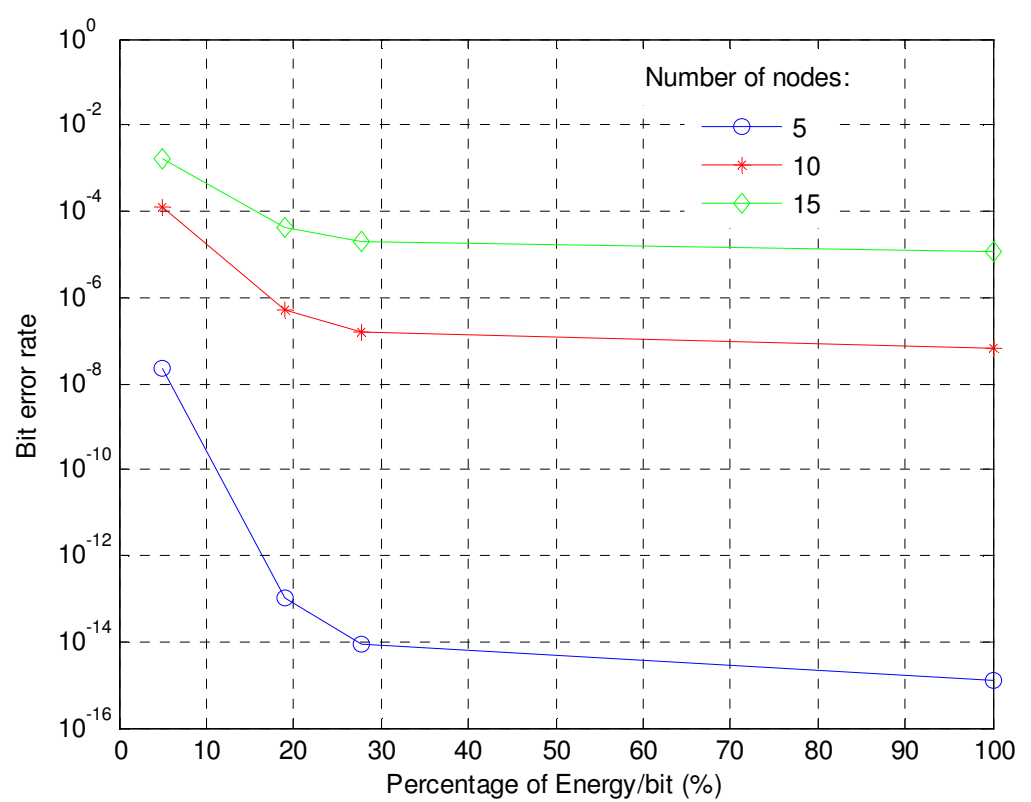

Fig. 9. BER versus percentage of energy per bit for the proposed DPCA with the number of active nodes of 5,10 and 15 .

\section{CONCLUSIONS}

In this work we have investigated the utilization of distributed power control algorithm (DPCA) as mechanism to increase the energy efficiency in OCDMA access network with noncoherent 2-D codes. In our proposed scheme, based on the classical DPCA, we have modeled the energy efficiency considering optical fiber transmission and network infrastructure as encoders, decoders and star coupler. This model confirms that the energy consumption of the network infrastructure is larger than the energy consumption of the transmission infrastructure for our numerical example. In the proposed scheme it is possible to obtain the energy efficiency according to the level of BER and the quality of service (QoS) requirements. The obtained results have shown economy of $70 \%$ in the transmitted energy per bit, at the penalty of one order of magnitude of BER.

In the proposed scheme it was possible to define the energy efficiency according to the BER level accomplished the quality of service (QoS) requirements.

\section{APPENDIX}

The derivation of (18) is obtained considering that the optimum solution for the power allocation problem satisfies the following set of equations [21]: 


$$
p_{i}^{*}=\sum_{j \neq i}^{K} h_{i j} p_{j}^{*}+\eta_{i}, \quad i=1, . ., K
$$

where $\eta_{i}=\gamma_{i}^{*} \sigma^{2} / g_{i i}$. Now, if $z(x)$ is an arbitrary odd function that has null value only at origin, equation (A.1) is the only equilibrium point of the following continuous-time dynamical system [19][21]:

$$
\dot{p}_{i}=z\left(-p_{i}+\sum_{j \neq i}^{K} h_{i j} p_{j}+\eta_{i}\right), \quad i=1, . ., K
$$

This control scheme is general and constitutes a systematic form to describe the dynamical behavior of various DPCAs. The sigmoidal [21], the Foschini [19], and recently the Verhulst [14] algorithm can be seen as particular structures of the general form. In the interval $t$ to $t+$ $\alpha_{i}$, the secant line to the curve $p_{i}(t)$ is indistinguishable from the tangent line in $t$ for small values of $\alpha_{i}$ if $p_{i}(t)$ is a continuous curve. In this case, we can write the approximation:

$$
p_{i}\left(t+\alpha_{i}\right) \approx p_{i}(t)+\alpha_{i} z_{i}\left(-p_{i}(t)+\sum_{j \neq i}^{K} h_{i j} p_{j}(t)+\eta_{i}\right)
$$

for $\alpha_{i}>0, i=1, \ldots, K$, which is the well-known Euler integration method. From the above exposition, the sequence $p_{i}[n]=p_{1}\left(n \alpha_{1}\right)$, can be recursively obtained by:

$$
p_{i}\left[n+\alpha_{i}\right]=p_{i}[n]+\alpha_{i} z_{i}\left(-p_{i}[n]+\sum_{j \neq i}^{K} h_{i j} p_{j}[n]+\eta_{i}\right)
$$

for $i=1, \ldots, K$. From definitions in (8) and (14) results:

$$
-p_{i}+\sum_{j \neq i}^{K} h_{i j} p_{j}+\eta_{i}=-\left(1-\frac{\gamma_{i}^{*}}{\gamma_{i}}\right) p_{i} \quad i=1, . ., K
$$

The DPCA developed by Foschini and Miljanic [19] and illustrated by (18), can be stated defining: $z_{i}(x)=x, \alpha i=\alpha, i=1, \ldots, K$.

\section{REFERENCES}

[1] K. Hinton, J. Baliga, M. Z. Feng, R.W.A. Ayre and R. S. Tucker, "Power consumption and energy efficiency in the internet”. IEEE Network, vol. 25, no. 2, pp. 6-12, March/April 2011.

[2] S. Yoo, "Energy Efficiency in the Future Internet: the Role of Optical Packet Switching and Optical Label Switching", IEEE J Selected Topics in Quantum Electronics, vol. 17, no. 2, pp. 406 - 418, March-April 2011.

[3] Rodney S. Tucker, "Green Optical Communications - Part Iः Energy Limitations in Transport", IEEE J Selected Topics in Quantum Electronics, vol. 17, no. 2, pp. 245 - 260, March-April 2011.

[4] Rodney S. Tucker, "Green Optical Communications - Part II: Energy Limitations in Networks", IEEE J Selected Topics in Quantum Electronics, vol. 17, no. 2, pp. 261 - 274, March-April 2011.

[5] A. Stok and E. H. Sargent, "Lighting the local area: Optical codedivision multiple-access and quality of service provisioning," IEEE Network, vol. 14, no. 6, pp. 42-46, Nov./Dec. 2000.

[6] G.-C. Yang and W.C. Kwong, Prime codes with applications to CDMA optical and wireless networks, Artech House, Boston, MA, 2002. 
[7] K. Fouli and M. Maier, "Ocdma and optical coding: Principles, applications, and challenges," IEEE Communications Magazine, vol. 45, no. 8, pp. 27-34, August 2007.

[8] H. Yin and D. J. Richardson, Optical code division multiple access communication networks: theory and applications. Berlin: Springer-Verlag and Tsinghua University Press, 2009.

[9] F R. Durand, L. Galdino, L. H. Bonani, F. R. Barbosa, M. L. F. Abbade and Edson Moschim, "The Effects of Polarization Mode Dispersion on 2D Wavelength-Hopping Time Spreading Code Routed Networks", Photonics Network Communications, vol. 20, no. 1, pp. 27 - 32, Aug. 2010. DOI 10.1007/s11107-0100242-6.

[10] N. Tarhuni, T. Korhonen, M. Elmusrati and E. Mutafungwa, "Power Control of Optical CDMA Star Networks", Optics Communications, vol. 259, pp. 655 - 664, Mar. 2006.

[11] E. Inaty, H. Shalaby, P. Fortie, and L. Rusch, Multirate "Optical Fast Frequency Hopping CDMA System Using Power Control”, Journal of Lightwave Tech., vol. 20, n. 2, pp.166 - 177, March 2003.

[12] F. R. Durand and T. Abrão, "Distributed SNIR Optimization Based on the Verhulst Model in Optical Code Path Routed Networks With Physical Constraints", Journal of Optical Communications and Networking, vol. 3, no. 9, pp. 683-691, Sep. 2011. doi:10.1364/JOCN.3.000683

[13] F. R. Durand, M. S. Filho and T. Abrão, "The effects of power control on the optical CDMA random access protocol", Optical Switching and Networking, (In press)～doi:10.1016/j.osn.2011.06.002

[14] T. J. Gross, T. Abrão and P. J. E. Jeszensky. "Distributed power control algorithm for multiple access systems based on Verhulst model”. AEU -- International Journal of Electronics and Communications. vol. 65, n.4, April 2011, pp. 361-372.

[15] Y. Huang, V. Baby, I. Glesk, C. Bres, C. Greiner, D. Iazikov, T. Mossberg, and P. Prucnal, "Novel multicode-processing platform for wavelength-hopping time-spreading optical cdma: A path to device miniaturization and enhanced network functionality," IEEE Journal of Selected Topics in Quantum Electronics, vol. 13, no. 5, pp. 1471-1479, Sept./Oct. 2007.

[16] C. Brès and P. Prucnal, "Code-empowered lightwave networks," Journal of Lightwave Technology, vol. 25, no. 10, pp. 2911-2921, Oct. 2007.

[17] K. O. Hill and G. Meltz, "Fiber bragg grating technology fundamentals and overview", Journal of Lightwave Technology, vol. 15, no. 8, pp. 1263-1276, Aug. 1997.

[18] D. Goodman and Narayan Mandayan "Power control for wireless data", IEEE Personal Communications, vol. 7, no. 2, pp. 48 - 54, April 2000.

[19] G. Foschini and Z. Miljanic, "A simple distributed autonomous power control algorithm and its convergence”, IEEE Trans. Veh. Technol., vol. 42, n. 4, pp. 641-646, Nov. 1993.

[20] Core Optics Ultra-FEC Chip. (2010). [Online]. Available: http://www.coreoptics.com/

[21] Z. Uykan and H. Koivo, "Sigmoid-basis nonlinear power-control algorithm for mobile radio systems", IEEE Trans. Veh. Technol. vol. 53, n.1, pp. 265-271, Jan 2004. 